APPLICATIONES MATHEMATICAE

27,3 (2000), pp. 319-333

P. B. MUCHA and W. ZAJA̧CZKOWSKI (Warszawa)

\title{
ON LOCAL EXISTENCE OF SOLUTIONS OF THE FREE BOUNDARY PROBLEM FOR AN INCOMPRESSIBLE VISCOUS SELF-GRAVITATING FLUID MOTION
}

Abstract. The local-in-time existence of solutions of the free boundary problem for an incompressible viscous self-gravitating fluid motion is proved. We show the existence of solutions with lowest possible regularity for this problem such that $u \in W_{r}^{2,1}\left(\widetilde{\Omega}^{T}\right)$ with $r>3$. The existence is proved by the method of successive approximations where the solvability of the CauchyNeumann problem for the Stokes system is applied. We have to underline that in the $L_{p}$-approach the Lagrangian coordinates must be used. We are looking for solutions with lowest possible regularity because this simplifies the proof and decreases the number of compatibility conditions.

1. Introduction. In this paper we consider the motion of a viscous incompressible fluid in a bounded domain $\Omega_{t} \subset \mathbb{R}^{3}$ with a free boundary $S_{t}$ which is under the self-gravitational force. Let $v=v(x, t)$ be the velocity of the fluid, $p=p(x, t)$ the pressure, $\nu$ the constant viscosity coefficient and $p_{0}$ the external pressure. Then the problem is described by the following system:

$$
\begin{array}{ll}
v_{t}+v \cdot \nabla v-\operatorname{div} \mathbb{T}(v, p)=\nabla U & \text { in } \widetilde{\Omega}^{T}, \\
\operatorname{div} v=0 & \text { in } \widetilde{\Omega}^{T}, \\
\mathbb{T}(u, p) \cdot \bar{n}=-p_{0} \bar{n} & \text { on } \widetilde{S}^{T}, \\
\left.v\right|_{t=0}=v_{0} & \text { in } \Omega, \\
\left.\Omega_{t}\right|_{t=0}=\Omega,\left.S_{t}\right|_{t=0}=S, & \\
v \cdot \bar{n}=-\varphi_{t} /|\nabla \varphi| & \text { on } \widetilde{S}^{T},
\end{array}
$$

2000 Mathematics Subject Classification: 35Q30, 76D05.

Key words and phrases: local existence, Navier-Stokes equations, incompressible viscous barotropic self-gravitating fluid, sharp regularity, anisotropic Sobolev space.

Research supported by Polish KBN Grant 2 P03A 03816. 
where $\widetilde{\Omega}^{T}=\bigcup_{t \leq T} \Omega_{t} \times\{t\}, \widetilde{S}^{T}=\bigcup_{t \leq T} S_{t} \times\{t\}, \varphi(x, t)=0$ describes $S_{t}$ at least locally, $\bar{n}$ is the unit outward vector normal to $S_{t}, \bar{n}=\nabla \varphi /|\nabla \varphi|, \Omega_{t}$ is the domain at time $t, S_{t}=\partial \Omega_{t}, t \leq T$. Moreover, the dot $\cdot$ denotes the scalar product in $\mathbb{R}^{3}$.

By $\mathbb{T}=\mathbb{T}(v, p)$ we denote the stress tensor of the form

$$
\mathbb{T}(v, p)=\left\{T_{i j}\right\}_{i, j=1,2,3}=\left\{-p \delta_{i j}+D_{i j}(v)\right\}_{i, j=1,2,3}
$$

where

$$
\mathbb{D}(v)=\left\{D_{i j}(v)\right\}_{i, j=1,2,3}=\left\{\nu\left(v_{i, x_{j}}+v_{j, x_{i}}\right)\right\}_{i, j=1,2,3}
$$

is the velocity deformation tensor.

Moreover, $U\left(\Omega_{t}, x, t\right)$ is the self-gravitational potential

$$
U\left(\Omega_{t}, x, t\right)=k \int_{\Omega_{t}} \frac{d y}{|x-y|},
$$

where $k$ is the gravitation constant and some arguments of $U$ are omitted in evident cases.

In view of the equation $(1.1)_{2}$ and the kinematic condition $(1.1)_{6}$ the total volume is conserved:

$$
\left|\Omega_{t}\right|=\int_{\Omega_{t}} d x=\int_{\Omega} d x=|\Omega| .
$$

Let $\Omega$ be given. Then we introduce the Lagrangian coordinates $\xi$ as the initial data for the following Cauchy problem:

$$
\frac{\partial x}{\partial t}=v(x, t),\left.\quad x\right|_{t=0}=\xi, \quad \xi=\left(\xi_{1}, \xi_{2}, \xi_{3}\right) .
$$

Integrating (1.6), we obtain a transformation which connects the Eulerian $x$ and the Lagrangian $\xi$ coordinates,

$$
x=x(\xi, t) \equiv \xi+\int_{0}^{t} u\left(\xi, t^{\prime}\right) d t^{\prime} \equiv x_{u}(\xi, t),
$$

where $u(\xi, t)=v\left(x_{u}(\xi, t), t\right)$ and the index $u$ in $x_{u}(\xi, t)$ will be omitted when no confusion can arise.

Then from $(1.1)_{6}$ we have $\Omega_{t}=\left\{x \in \mathbb{R}^{3}: x=x(\xi, t), \xi \in \Omega\right\}$ and $S_{t}=\left\{x \in \mathbb{R}^{3}: x=x(\xi, t), \xi \in S=S_{0}=\partial \Omega\right\}$.

Our aim is to prove the local-in-time existence of solutions to problem (1.1) with lowest possible regularity. Therefore we apply the $L_{p}$-approach. The result of the paper is the following theorem.

TheOREM 1.1. Let $r>3, v_{0} \in W_{r}^{2-2 / r}(\Omega), S \in W_{r}^{2-1 / r}$. Then there exists $T_{0}>0$ such that for all $T \leq T_{0}$ there exists a unique solution $(u, p)$ of (1.1) such that $u \in W_{r}^{2,1}\left(\widetilde{\Omega}^{T}\right), p \in W_{r}^{1,0}\left(\widetilde{\Omega}^{T}\right) \cap W_{r}^{1-1 / r, 1 / 2-1 /(2 r)}\left(\widetilde{S}^{T}\right)$ 
and the following estimate holds:

$$
\begin{aligned}
\|u\|_{W_{r}^{2,1}\left(\widetilde{\Omega}^{T}\right)}+\|p\|_{W_{r}^{1,0}\left(\widetilde{\Omega}^{T}\right)}+\|p\|_{W_{r}^{1-1 / r, 1 / 2-1 /(2 r)}\left(\widetilde{S}^{T}\right)} & \leq c(T)\left\|v_{0}\right\|_{W_{r}^{2-2 / r}(\Omega)^{2}} .
\end{aligned}
$$

To prove Theorem 1.1 we need solvability of the Cauchy-Neumann problem for the Stokes system from [3]. To recall the result we formulate the problem

$$
\begin{array}{ll}
u_{t}-\operatorname{div} \mathbb{T}(u, p)=F & \text { in } \Omega^{T}, \\
\operatorname{div} u=G & \text { in } \Omega^{T}, \\
\bar{n} \cdot \mathbb{T}(u, p)=H & \text { on } S^{T}, \\
\left.u\right|_{t=0}=u_{0} & \text { in } \Omega,
\end{array}
$$

where $\Omega^{T}=\Omega \times[0, T]$ and $S^{T}=S \times[0, T]$.

Theorem 1.2 (see [3]). Let $r>3, F \in L_{r}\left(\Omega^{T}\right), G \in W_{r}^{1,0}\left(\Omega^{T}\right)$,

$$
\begin{gathered}
G_{t}-\operatorname{div} F=\operatorname{div} B+A, \quad A, B \in L_{r}\left(\Omega^{T}\right), \\
H \in W_{r}^{1-1 / r, 1 / 2-1 /(2 r)}\left(S^{T}\right), \quad u_{0} \in W_{r}^{2-2 / r}(\Omega), \quad S \in W_{r}^{2-2 / r},
\end{gathered}
$$

and assume the compatibility conditions

$$
\operatorname{div} u_{0}=G(x, 0),\left.\quad \bar{n} \cdot \mathbb{T}\left(u_{0}, p_{0}\right)\right|_{S}=H(x, 0),
$$

where $p_{0}=\left.p\right|_{t=0}$. Then there exists a unique solution $(u, p)$ to problem (1.9) such that

$$
u \in W_{r}^{2,1}\left(\Omega^{T}\right), \quad p \in W_{r}^{1,0}\left(\Omega^{T}\right) \cap W_{r}^{1-1 / r, 1 / 2-1 /(2 r)}\left(S^{T}\right)
$$

and the following estimate holds:

$$
\begin{aligned}
& \|u\|_{W_{r}^{2,1}\left(\Omega^{T}\right)}+\|p\|_{W_{r}^{1,0}\left(\Omega^{T}\right)}+\|p\|_{W_{r}^{1-1 / r, 1 / 2-1 /(2 r)}\left(S^{T}\right)} \\
& \leq C(T)\left[\|F\|_{L_{r}\left(\Omega^{T}\right)}+\|G\|_{W_{r}^{1,0}\left(\Omega^{T}\right)}+\|B\|_{L_{r}\left(\Omega^{T}\right)}+\|A\|_{L_{r}\left(\Omega^{T}\right)}\right. \\
& \left.\quad+\|H\|_{W_{r}^{1-1 / r, 1 / 2-1 /(2 r)}\left(S^{T}\right)}+\left\|u_{0}\right\|_{W_{r}^{2-2 / r}(\Omega)}\right],
\end{aligned}
$$

where $C(T)$ is a constant increasing with $T$ which does not depend on the solution $(u, p)$.

Problem (1.1) without the self-gravitation force is considered in [4]. Moreover we recall that the local existence of solutions to problem (1.1) with surface tension is shown in [5].

2. Notation. We need the anisotropic Sobolev spaces $W_{r}^{m, n}\left(Q_{T}\right)$ where $m, n \in \mathbb{R}_{+} \cup\{0\}, r \geq 1$ and $Q_{T}=Q \times(0, T)$, with the norm 


$$
\begin{aligned}
& \|u\|_{W_{r}^{m, n}}^{r}\left(Q_{T}\right) \\
& =\int_{0}^{T} \int_{Q}|u(x, t)|^{r} d x d t \\
& +\sum_{0 \leq\left|m^{\prime}\right| \leq[|m|]} \int_{0}^{T} \int_{Q}\left|D_{x}^{m^{\prime}} u(x, t)\right|^{r} d x d t \\
& +\sum_{\left|m^{\prime}\right|=[|m|]} \int_{0}^{T} d t \int_{Q} \int_{Q} \frac{\left|D_{x}^{m^{\prime}} u(x, t)-D_{x}^{m^{\prime}} u\left(x^{\prime}, t\right)\right|^{r}}{\left|x-x^{\prime}\right|^{s+r(|m|-[|m|])}} d x d x^{\prime} \\
& +\sum_{0 \leq\left|n^{\prime}\right| \leq[|n|]} \int_{0}^{T} \int_{Q}\left|D_{t}^{n^{\prime}} u(x, t)\right|^{r} d x d t \\
& +\int_{Q} d x \int_{0}^{T} \int_{0}^{T} \frac{\left|D_{t}^{[n]} u(x, t)-D_{t}^{[n]}\left(x, t^{\prime}\right)\right|^{r}}{\left|t-t^{\prime}\right|^{1+r(n-[n])}} d t d t^{\prime},
\end{aligned}
$$

where $s=\operatorname{dim} Q,[\alpha]$ is the integral part of $\alpha, D_{x}^{l}=\partial_{x_{1}}^{l_{1}} \ldots \partial_{x_{s}}^{l_{s}}$ where $l=\left(l_{1}, \ldots, l_{s}\right)$ is a multiindex.

In the proof we will use the following results.

Proposition 2.1 (see [1]). Let $u \in W_{r}^{m, n}\left(\Omega_{T}\right), m, n \in \mathbb{R}_{+}$. If $q \geq r$ and

$$
\kappa=\sum_{i=1}^{3}\left(\alpha_{i}+\frac{1}{r}-\frac{1}{q}\right) \frac{1}{m}+\left(\beta+\frac{1}{r}-\frac{1}{q}\right) \frac{1}{n}<1
$$

then

$$
\left\|D_{t}^{\beta} D_{x}^{\alpha} u\right\|_{L_{q}\left(\Omega_{T}\right)} \leq \varepsilon^{1-\kappa}\|u\|_{W_{r}^{m, n}\left(\Omega_{T}\right)}+c \varepsilon^{-\kappa}\|u\|_{L_{r}\left(\Omega_{T}\right)}
$$

for all $\varepsilon \in(0,1)$.

Proposition 2.2 (see $[1,2]$ ). Let $u \in W_{r}^{2 m, m}\left(\Omega_{T}\right), m \in \mathbb{R}_{+}$. If $2 m-$ $1 / r>0$ then $\bar{u}=\left.u\right|_{S_{T}}$ is well defined as a function in $W_{r}^{2 m-1 / r, m-1 /(2 r)}\left(S_{T}\right)$ and

$$
\|\bar{u}\|_{W_{r}^{2 m-1 / r, m-1 /(2 r)}\left(S_{T}\right)} \leq c\|u\|_{W_{r}^{2 m, m}\left(\Omega_{T}\right)} .
$$

Proposition 2.3 (see $[1,2]$ ). Let $u \in W_{r}^{2 m-1 / r, m-1 /(2 r)}\left(S_{T}\right), m \in \mathbb{R}_{+}$. If $2 m-1 / r>0$ then there exists a function $\widetilde{u} \in W_{r}^{2 m, m}\left(\Omega_{T}\right)$ such that $\left.\widetilde{u}\right|_{S_{T}}=u$ and the following estimate holds:

$$
\|\widetilde{u}\|_{W_{r}^{2 m, m}\left(\Omega_{T}\right)} \leq c\|u\|_{W_{r}^{2 m-1 / r, m-1 /(2 r)}\left(S_{T}\right)} .
$$

In our considerations we will use well known imbedding theorems for Sobolev spaces. All constants are denoted by the same letter $c$. 
3. Proof of Theorem 1.1. To prove local existence of solutions to problem (1.1) we write it in the Lagrangian coordinates:

$$
\begin{array}{ll}
u_{t}-\operatorname{div}_{u} \mathbb{T}_{u}(u, q)=\nabla_{u} U_{u} & \text { in } \Omega^{T}, \\
\operatorname{div}_{u} u=0 & \text { in } \Omega^{T}, \\
\bar{n}_{u} \cdot \mathbb{T}_{u}(u, q)=-p_{0} \bar{n}_{u} & \text { on } S^{T}, \\
\left.u\right|_{t=0}=v_{0} & \text { on } \Omega,
\end{array}
$$

where $u(\xi, t)=v(x(\xi, t), t), q(\xi, t)=p(x(\xi, t), t), \nabla_{u}=\xi_{i, x} \nabla_{\xi_{i}}, \mathbb{T}_{u}(u, q)=$ $\mathbb{D}_{u}(u)-q I, \mathbb{D}_{u}(u)=\nu\left\{\xi_{k, x_{i}} u_{j, \xi_{k}}+\xi_{k, x_{j}} u_{i, \xi_{k}}\right\}_{i, j=1,2,3}$,

$$
U_{u}(\xi, t)=\int_{\Omega} \frac{k J_{y\left(\xi^{\prime}, t\right)} d \xi^{\prime}}{\left|x(\xi, t)-y\left(\xi^{\prime}, t\right)\right|},
$$

where $J_{x(\xi, t)}$ is the Jacobian of the transformation $x=x(\xi, t), \operatorname{div}_{u} u=$ $\xi_{k, x_{i}} u_{i, x_{k}}, \bar{n}_{u}(\xi, t)=\bar{n}(x(\xi, t), t), I$ is the unit matrix and the summation convention over repeated indices is used.

To prove the existence of solutions to (3.1) we use the following method of successive approximations:

$$
\begin{array}{ll}
u_{m+1, t}-\operatorname{div}_{u_{m}} \mathbb{T}_{u_{m}}\left(u_{m+1}, q_{m+1}\right)=\nabla_{u_{m}} U_{u_{m}} & \text { in } \Omega^{T}, \\
\operatorname{div}_{u_{m}} u_{m+1}=0 & \text { in } \Omega^{T}, \\
\bar{n}_{u_{m}} \cdot \mathbb{T}_{u_{m}}\left(u_{m+1}, q_{m+1}\right)=-p_{0} \bar{n}_{u_{m}} & \text { on } S^{T}, \\
\left.u_{m+1}\right|_{t=0}=v_{0} & \text { on } \Omega,
\end{array}
$$

where $m=0,1,2, \ldots$ and $u_{m}$ is treated as a given function. Assume $u_{0}=0$ and $q_{0}=0$.

To apply Theorem 1.2 we write (3.2) in the form

$$
\begin{array}{ll}
u_{m+1, t}-\operatorname{div} \mathbb{T}\left(u_{m+1}, q_{m+1}\right) & \\
= & \operatorname{div}_{u_{m}} \mathbb{T}_{u_{m}}\left(u_{m+1}, q_{m+1}\right) \\
\quad-\operatorname{div} \mathbb{T}\left(u_{m+1}, q_{m+1}\right)+\nabla_{u_{m}} U_{u_{m}} & \text { in } \Omega^{T}, \\
\operatorname{div} u_{m+1}=\operatorname{div} u_{m+1}-\operatorname{div}_{u_{m}} u_{m+1} & \text { in } \Omega^{T}, \\
\bar{n}_{0} \cdot \mathbb{T}\left(u_{m+1}, q_{m+1}\right) & \\
=\bar{n}_{0} \cdot \mathbb{T}\left(u_{m+1}, q_{m+1}\right) & \\
\quad-\bar{n}_{u_{m}} \cdot \mathbb{T}_{u_{m}}\left(u_{m+1}, q_{m+1}\right)-p_{0} \bar{n}_{u_{m}} & \text { on } S^{T}, \\
\left.u_{m+1}\right|_{t=0}=v_{0} & \text { on } \Omega,
\end{array}
$$

where the operators without index contain derivatives with respect to $\xi$ and $\bar{n}_{0}$ is the unit outward vector normal to $S$. 
First we obtain a uniform bound for the sequence $\left\{u_{m}\right\}_{m=0}^{\infty}$ determined by $(3.3)$.

Lemma 3.1. Assume that $S \in W_{r}^{2-1 / r}, v_{0} \in W_{r}^{2-2 / r}(\Omega)$. Then

$$
\begin{aligned}
\left\|u_{m}\right\|_{W_{r}^{2,1}\left(\Omega_{T}\right)}+\left\|q_{m}\right\|_{W_{r}^{1,0}\left(\Omega^{T}\right)}+ & \left\|q_{m}\right\|_{W_{r}^{1-1 / r, 1 / 2-1 /(2 r)}\left(S^{T}\right)} \\
& \leq c\left(\left\|v_{0}\right\|_{W_{r}^{2-2 / r}(\Omega)},\|S\|_{W_{r}^{2-2 / r}}\right)
\end{aligned}
$$

if $T$ is small enough.

Pr o of. Applying Theorem 1.2 to problem (3.3) yields

$$
\begin{aligned}
&\left\|u_{m+1}\right\|_{W_{r}^{2,1}\left(\Omega_{T}\right)}+\left\|q_{m+1}\right\|_{W_{r}^{1,0}\left(\Omega^{T}\right)}+\left\|q_{m+1}\right\|_{W_{r}^{1-1 / r, 1 / 2-1 /(2 r)}\left(S^{T}\right)} \\
& \leq c\left\|\operatorname{div} \mathbb{T}\left(u_{m+1}, q_{m+1}\right)-\operatorname{div}_{u_{m}} \mathbb{T}_{u_{m}}\left(u_{m+1}, q_{m+1}\right)\right\|_{L_{r}\left(\Omega^{T}\right)} \\
&+c\left\|\nabla_{u_{m}} U_{u_{m}}\right\|_{L_{r}\left(\Omega^{T}\right)}+c\left\|\operatorname{div} u_{m+1}-\operatorname{div}_{u_{m}} u_{m+1}\right\|_{W_{r}^{1,0}\left(\Omega^{T}\right)} \\
&+c \| \bar{n}_{0} \cdot \mathbb{T}\left(u_{m+1}, q_{m+1}\right) \\
&-\bar{n}_{u_{m}} \cdot \mathbb{T}_{u_{m}}\left(u_{m+1}, q_{m+1}\right) \|_{W_{r}^{1-1 / r, 1 / 2-1 /(2 r)}\left(S^{T}\right)} \\
&+c\left\|\bar{n}_{u_{m}}\right\|_{W_{r}^{1-1 / r, 1 / 2-1 /(2 r)}\left(S^{T}\right)}+c\left\|v_{0}\right\|_{W_{r}^{2-2 / r}(\Omega)} \\
&+c\left\|\left(\left(I-A^{*}\left(u_{m}\right)\right) u_{m+1}\right)_{t}\right\|_{L_{r}\left(\Omega^{T}\right)},
\end{aligned}
$$

where $\left(\left(I-A^{*}\left(u_{m}\right)\right) u_{m+1}\right)_{t}$ is treated as $\widetilde{B}$ from Theorem $1.2(\widetilde{A}=0)$ and $A_{i j}\left(u_{m}\right)=\delta_{i j}+\int_{0}^{t} u_{m i, \xi_{j}} d \tau, A_{k l}^{*}\left(u_{m}\right)=A_{l k}^{-1}\left(u_{m}\right)$. Here we note that

$$
\operatorname{div}_{u_{m}} u_{m+1}=A_{k l}^{-1} \partial_{\xi_{l}} u_{m+1}^{l}=\operatorname{div}_{\xi}\left(A^{*} u_{m+1}\right),
$$

which follows from $\sum_{k=1}^{3} \frac{\partial}{\partial \xi_{k}} A_{l k}\left(u_{m}\right)(\xi, t)=0$. All the above relations hold under the assumption that $\operatorname{div}_{u_{m-1}} u_{m}=0$.

To continue the induction we need to have $\operatorname{div}_{u_{m}} u_{m+1}=0$, but this is given by $(3.3)_{2}$.

Now we estimate the particular terms from the r.h.s. of (3.5). Define $a_{m}=T^{(r-1) / r}\left\|u_{m}\right\|_{W_{r}^{2,1}\left(\Omega^{T}\right)}, \alpha_{m}(t)=\left\{\alpha_{i j}\left(u_{m}\right)\right\}=\left\{\int_{0}^{t} u_{m i, \xi_{j}} d \tau\right\}$.

For $r>3$ we have $\left\|\alpha_{m}\right\|_{L_{\infty}(\Omega)} \leq c a_{m}$.

To estimate the first term on the r.h.s. of (3.5) we calculate

$$
\begin{aligned}
\operatorname{div}_{u_{m}} & \mathbb{T}_{u_{m}}\left(u_{m+1}, q_{m+1}\right)-\operatorname{div} \mathbb{T}\left(u_{m+1}, q_{m+1}\right) \\
= & \left\{\nu\left(\xi_{l x_{j}} \xi_{k x_{j} x_{s}} x_{s \xi_{l}} \delta_{\sigma i}+\xi_{l x_{j}} \xi_{k x_{i} x_{s}} x_{s \xi_{l}} \delta_{\sigma j}\right) u_{m+1 \sigma, \xi_{k}}\right. \\
& +\nu\left(\xi_{l x_{i}} \xi_{k x_{j}}-\delta_{j k} \delta_{j l}\right) u_{m+1 i, \xi_{l} \xi_{k}}+\nu\left(\xi_{l x_{j}} \xi_{k \xi_{i}}-\delta_{l j} \delta_{k i}\right) u_{m+1 j, \xi_{l} \xi_{k}} \\
& \left.-\left(\xi_{l x_{j}}-\delta_{l j} q_{m+1, \xi_{l}}\right)\right\},
\end{aligned}
$$

where the matrix $\xi_{, x}$ depends on $u_{m}$. 
Since $x_{i \xi_{j}}=\delta_{i j}+\int_{0}^{t} u_{i \xi_{j}}(\tau) d \tau=\delta_{i j}+\alpha_{i j}$ and $\xi_{j x_{i}}$ is the inverse matrix to $x_{i \xi_{j}}$, we have

$$
\xi_{j x_{i}}=\delta_{i j}+\phi_{i j}(\alpha),
$$

where $\phi_{i j}$ is a polynomial matrix-valued function which contains terms of $\alpha$ and $\alpha^{2}\left(\alpha=\left\{\alpha_{i j}\right\}\right)$. Then $\xi_{j, x_{i} x_{k}}=\phi_{i j, \alpha_{r s}} \alpha_{r s, \xi_{\sigma}} \xi_{\sigma, x_{k}}$, where $\alpha_{r s, \xi_{\sigma}}=$ $\int_{0}^{t} u_{r, \xi_{s} \xi_{\sigma}}(\tau) d \tau$.

Then we write the first term of the r.h.s. of (3.5) in the form

$$
\begin{array}{r}
\left\|\psi_{1}\left(\alpha_{m}\right)\left(I-A\left(u_{m}\right)\right)\left(u_{m+1, \xi \xi}+q_{m+1, \xi}\right)+\psi_{2}\left(\alpha_{m}\right) A\left(u_{m}\right)_{, \xi} u_{m+1, \xi}\right\|_{L_{r}\left(\Omega^{T}\right)} \\
\leq \phi\left(a_{m}\right) a_{m}\left(\left\|u_{m+1}\right\|_{W_{r}^{2,1}\left(\Omega^{T}\right)}+\left\|q_{m+1}\right\|_{W_{r}^{1,0}\left(\Omega^{T}\right)}\right),
\end{array}
$$

where $\psi_{i}$ are some functions with $\psi_{i}(0) \neq 0$ and $\phi$ always denotes an increasing positive function.

We estimate the third term by the same quantity.

The fourth term can be expressed in the form

$$
\begin{aligned}
\| \psi_{3}\left(\alpha_{m}\right) \alpha_{m} u_{m+1, \xi} & +\psi_{4}\left(\alpha_{m}\right) \alpha_{m} q_{m+1} \|_{W_{r}^{1-1 / r, 1 / 2-1 /(2 r)}\left(S^{T}\right)} \\
\leq & \left\|\psi_{3}\left(\alpha_{m}\right) \alpha_{m} u_{m+1, \xi}\right\|_{W_{r}^{1-1 / r, 1 / 2-1 /(2 r)}\left(S^{T}\right)} \\
& +\left\|\psi_{4}\left(\alpha_{m}\right) \alpha_{m} q_{m+1}\right\|_{W_{r}^{1-1 / r, 1 / 2-1 /(2 r)}\left(S^{T}\right)} \equiv I+J,
\end{aligned}
$$

where

$$
\begin{aligned}
I \leq & \left(\int_{0}^{T}\left\|\psi_{3}\left(\alpha_{m}\right) \alpha_{m} u_{m+1, \xi}\right\|_{W_{r}^{1}(\Omega)}^{r} d \tau\right)^{1 / r} \\
& +\left(\int_{\Omega}\left\|\psi_{3}\left(\alpha_{m}\right) \alpha_{m} u_{m+1, \xi}\right\|_{W_{r}^{1 / 2}(0, T)}^{r}\right)^{1 / r} \equiv L+K .
\end{aligned}
$$

Next we have

$$
\begin{aligned}
L \leq & \left(\int_{0}^{T}\left\|\psi_{3}\left(\alpha_{m}\right) \alpha_{m} u_{m+1, \xi}\right\|_{L_{r}(\Omega)}^{r} d \tau\right)^{1 / r} \\
& +\left(\int_{0}^{T}\left\|\psi_{3, \alpha_{m}}\left(\alpha_{m}\right) \int_{0}^{t} u_{m, \xi \xi} d \tau \alpha_{m} u_{m+1, \xi}\right\|_{L_{r}(\Omega)}^{r} d t\right)^{1 / r} \\
& +\left(\int_{0}^{T}\left\|\psi_{3}\left(\alpha_{m}\right) \int_{0}^{t} u_{m, \xi \xi} d \tau u_{m+1, \xi}\right\|_{L_{r}(\Omega)}^{r} d t\right)^{1 / r} \\
& +\left(\int_{0}^{T}\left\|\psi_{3}\left(\alpha_{m}\right) \alpha_{m} u_{m+1, \xi \xi}\right\|_{L_{r}(\Omega)}^{r} d \tau\right)^{1 / r} \equiv L_{1}+L_{2}+L_{3}+L_{4} .
\end{aligned}
$$


Continuing, we have

$$
\begin{aligned}
L_{1} & \leq \phi\left(a_{m}(T)\right) a_{m}(T)\left\|u_{m+1}\right\|_{W_{r}^{2,1}\left(\Omega^{T}\right)} \\
L_{2}+L_{3} & \leq \phi\left(a_{m}(T)\right) a_{m}(T)\left(\int_{0}^{T}\left\|\int_{0}^{t} u_{m, \xi \xi} d \tau u_{m+1, \xi}\right\|_{L_{r}(\Omega)}^{r} d t\right)^{1 / r} \\
& \leq \phi\left(a_{m}(T)\right) a_{m}(T) a_{m}^{2}(T)\left\|u_{m+1}\right\|_{W_{r}^{2,1}\left(\Omega^{T}\right)} \\
L_{4} & \leq \phi\left(a_{m}(T)\right) a_{m}(T)\left\|u_{m+1}\right\|_{W_{r}^{2,1}\left(\Omega^{T}\right)} .
\end{aligned}
$$

Next we examine

$$
\begin{aligned}
K \leq & \left(\int_{\Omega} d \xi \int_{0}^{T} d t \int_{0}^{T} d t^{\prime} \frac{\left|\psi_{3}\left(\alpha_{m}(t)\right)-\psi_{3}\left(\alpha_{m}\left(t^{\prime}\right)\right)\right|^{r}\left|\alpha_{m}(t)\right|^{r}\left|u_{m+1, \xi}(t)\right|^{r}}{\left|t-t^{\prime}\right|^{1+r / 2}}\right)^{1 / r} \\
& +\left(\int_{\Omega} d \xi \int_{0}^{T} d t \int_{0}^{T} d t^{\prime} \frac{\left|\psi_{3}\left(\alpha_{m}\left(t^{\prime}\right)\right)\right|^{r}\left|\alpha_{m}(t)-\alpha_{m}\left(t^{\prime}\right)\right|^{r}\left|u_{m+1, \xi}(t)\right|^{r}}{\left|t-t^{\prime}\right|^{1+r / 2}}\right)^{1 / r} \\
& +\left(\int_{\Omega} d \xi \int_{0}^{T} d t \int_{0}^{T} d t^{\prime} \frac{\left|\psi_{3}\left(\alpha_{m}(t)\right)\right|^{r}\left|\alpha_{m}(t)\right|^{r}\left|u_{m+1, \xi}(t)-u_{m+1, \xi}\left(t^{\prime}\right)\right|^{r}}{\left|t-t^{\prime}\right|^{1+r / 2}}\right)^{1 / r} \\
\equiv & K_{1}+K_{2}+K_{3} .
\end{aligned}
$$

Using the formula

$$
\begin{aligned}
& \psi_{3}\left(\alpha_{m}(t)\right)-\psi_{3}\left(\alpha_{m}\left(\alpha_{m}\left(t^{\prime}\right)\right)\right. \\
& \quad=\left(\alpha_{m}(t)-\alpha_{m}\left(t^{\prime}\right)\right) \int_{0}^{1} \psi_{3, \alpha_{m}\left(t^{\prime}\right)}\left(\alpha_{m}\left(t^{\prime}\right)+s\left(\alpha_{m}(t)-\alpha_{m}\left(t^{\prime}\right)\right) d s\right.
\end{aligned}
$$

we obtain

$$
\begin{aligned}
& K_{1}+K_{2} \\
& \leq \phi\left(a_{m}(T)\right)\left(\int_{\Omega} d \xi \int_{0}^{T} d t \int_{0}^{T} d t^{\prime} \frac{\left|\int_{t^{\prime}}^{t} u_{m, \xi} d \tau\right|^{r}\left|u_{m+1, \xi}(t)\right|^{r}}{\left|t-t^{\prime}\right|^{1+r / 2}}\right)^{1 / r} \\
& \leq \phi\left(a_{m}(T)\right)\left(\int_{\Omega} d \xi \int_{0}^{T} d t \int_{0}^{T} d t^{\prime}\left|t-t^{\prime}\right|^{r / 2-2} \int_{t^{\prime}}^{t}\left|u_{m, \xi}\right|^{r} d \tau\left|u_{m+1, \xi}(t)\right|^{r}\right)^{1 / r} \\
& \leq \phi\left(a_{m}(T)\right)\left\|u_{m}\right\|_{W_{r}^{2,1}\left(\Omega^{T}\right)}\left(\int_{\Omega} d \xi \int_{0}^{T} d t \int_{0}^{T} d t^{\prime}\left|t-t^{\prime}\right|^{r / 2-2}\left|u_{m+1, \xi}\right|^{r}\right)^{1 / r} \equiv K_{4} .
\end{aligned}
$$

Integrating with respect to $t^{\prime}$ we get $(r / 2>1)$

$$
K_{4} \leq \phi\left(a_{m}(T)\right) T^{r / 2-1}\left\|u_{m}\right\|_{W_{r}^{2,1}\left(\Omega^{T}\right)}\left\|u_{m+1}\right\|_{W_{r}^{2,1}\left(\Omega^{T}\right)} .
$$


Finally

$$
K_{3} \leq \phi\left(a_{m}(T)\right) a_{m}(T)\left\|u_{m+1}\right\|_{W_{r}^{2,1}\left(\Omega^{T}\right)} .
$$

Summarizing the above considerations we obtain

$$
\begin{aligned}
I \leq & \phi\left(a_{m}(T)\right) a_{m}(T)\left\|u_{m+1}\right\|_{W_{r}^{2,1}\left(\Omega^{T}\right)} \\
& +\phi\left(a_{m}(T)\right) T^{r / 2-1}\left\|u_{m}\right\|_{W_{r}^{2,1}\left(\Omega^{T}\right)}\left\|u_{m+1}\right\|_{W_{r}^{2,1}\left(\Omega^{T}\right)} .
\end{aligned}
$$

Similarly, we obtain

$$
\begin{aligned}
J \leq & \phi\left(a_{m}(T)\right) a_{m}(T)\left\|\widetilde{q}_{m+1}\right\|_{W_{r}^{1,1 / 2}\left(\Omega^{T}\right)} \\
& +\phi\left(a_{m}(T)\right) T^{r / 2-1}\left\|u_{m}\right\|_{W_{r}^{2,1}\left(\Omega^{T}\right)}\left\|\widetilde{q}_{m+1}\right\|_{W_{r}^{1,1 / 2}\left(\Omega^{T}\right)},
\end{aligned}
$$

where $\widetilde{q}_{m+1}$ is an extension of $q_{m+1} \in W_{r}^{1-1 / r, 1 / 2-1 /(2 r)}\left(S^{T}\right)$.

The fifth term on the r.h.s. of (3.5) is estimated by

$$
\begin{aligned}
\left\|\psi_{5}\left(\alpha_{m}\right)\right\|_{W_{r}^{1-1 / r, 1 / 2-1 /(2 r)}\left(S^{T}\right)} \leq & \left(\int_{0}^{T}\left\|\psi_{5}\left(\alpha_{m}(t)\right)\right\|_{W_{r}^{1-1 / r}(S)} d t\right)^{1 / r} \\
& +\left(\int_{S}\left\|\psi_{5}\left(\alpha_{m}(t)\right)\right\|_{W_{r}^{1 / 2-1 /(2 r)}(0, T)} d \xi\right)^{1 / r} \\
\equiv & M_{1}+M_{2},
\end{aligned}
$$

where

$$
\begin{aligned}
& M_{1} \leq \phi\left(a_{m}(T)\right)\left(\int_{0}^{T} d t\left\|\int_{0}^{t} u_{m, \xi} d \tau\right\|_{W_{r}^{1}(\Omega)}^{r}\right)^{1 / r} \leq \phi\left(a_{m}(T)\right) T^{1 / r} a_{m}(T), \\
& M_{2} \leq\left(\int_{S} d \xi \int_{0}^{T} d t \int_{0}^{T} d t^{\prime} \frac{\left|\psi_{5}\left(\alpha_{m}(t)\right)-\psi_{5}\left(\alpha_{m}\left(t^{\prime}\right)\right)\right|^{r}}{\left|t-t^{\prime}\right|^{1+r(1 / 2-1 /(2 r))}}\right)^{1 / r}
\end{aligned}
$$

using (3.6) we have

$$
\begin{aligned}
\phi\left(a_{m}(T)\right) & \left(\int_{S} d \xi \int_{0}^{T} d t \int_{0}^{T} d t^{\prime} \frac{\left|\int_{t^{\prime}}^{t} u_{m, \xi}(\xi, \tau) d \tau\right|^{r}}{\left|t-t^{\prime}\right|^{1+r(1 / 2-1 /(2 r))}}\right)^{1 / r} \\
& \leq\left(\int_{S} d \xi \int_{0}^{T}\left|u_{m, \xi}(\xi, \tau)\right|^{r} d \tau\right)^{1 / r}\left(\int_{0}^{T} d t \int_{0}^{T}\left|t-t^{\prime}\right|^{r / 2-3 / 2} d t^{\prime}\right)^{1 / r} \\
& \leq \phi\left(a_{m}(T)\right) T^{1 / 2+r / 2}\left\|u_{m}\right\|_{W_{r}^{2,1}\left(\Omega^{T}\right)} .
\end{aligned}
$$

The seventh term of the r.h.s. of (3.5) will be considered in the from

$$
\begin{aligned}
\left\|\left(\left(I-A^{*}\left(u_{m}\right)\right) u_{m+1}\right)_{t}\right\|_{L_{r}\left(\Omega^{T}\right) \leq} \leq & \left\|\psi_{6}\left(\alpha_{m}\right) \alpha_{m} u_{m+1, t}\right\|_{L_{r}\left(\Omega^{T}\right)} \\
& +\left\|\psi_{7}\left(\alpha_{m}\right) u_{m, \xi} u_{m+1}\right\|_{L_{r}\left(\Omega^{T}\right)} \equiv N_{1}+N_{2}
\end{aligned}
$$


and we have

$$
\begin{aligned}
N_{1} \leq & \phi\left(a_{m}(T)\right)\left|\alpha_{m}\right|\left\|u_{m+1}\right\|_{W_{r}^{2,1}\left(\Omega^{T}\right)} \\
N_{2} \leq & \phi\left(a_{m}(T)\right)\left\|\left(u_{m, \xi}-v_{0, \xi}+v_{0, \xi}\right)\left(v_{0}+\int_{0}^{t} u_{m+1, t} d t\right)\right\|_{L_{r}\left(\Omega^{T}\right)} \\
\leq & \phi\left(a_{m}(T)\right) T^{1 / r}\left\|v_{0}\right\|_{W_{r}^{2-2 / r}(\Omega)}^{2} \\
& +\phi\left(a_{m}(T)\right)\left\|u_{m}\right\|_{W_{r}^{2,1}\left(\Omega^{T}\right)} a_{m+1} \\
& +\phi\left(a_{m}(T)\right)\left\|v_{0}\right\|_{W_{r}^{2-2 / r}(\Omega)} T^{\beta} \\
& \times\left(\left\|u_{m}\right\|_{W_{r}^{2,1}\left(\Omega^{T}\right)}+\left\|v_{0}\right\|_{W_{r}^{2-2 / r}(\Omega)}\right) .
\end{aligned}
$$

In the last term of the r.h.s. of $(3.7)_{2}$ we have applied the imbedding $W_{r}^{1,1 / 2}\left(\Omega^{T}\right) \subset C^{\beta}\left(0, T ; L_{r}(\Omega)\right)$ with $0<\beta<1 / 2-1 / r$. This enables us to get

$$
\left\|u_{m, \xi}-v_{0, \xi}\right\|_{L_{r}} \leq T^{\beta}\left(\left\|u_{m}\right\|_{W_{r}^{2,1}\left(\Omega^{T}\right)}+\left\|v_{0}\right\|_{W_{r}^{2-2 / r}(\Omega)}\right) .
$$

Finally we consider the second term of the r.h.s. of (3.5). We have

$$
\begin{aligned}
\nabla_{u_{m}} U_{u_{m}}= & \nabla_{u_{m}} \int_{\Omega} \frac{J_{x_{u_{m}}\left(\xi^{\prime}, t\right)}}{\left|x_{u_{m}}(\xi, t)-x_{u_{m}}\left(\xi^{\prime}, t\right)\right|} d \xi^{\prime} \\
= & -\int_{\Omega} \frac{\nabla_{u_{m}} x_{u_{m}}(\xi, t) \cdot\left(x_{u_{m}}(\xi, t)-x_{u_{m}}\left(\xi^{\prime}, t\right)\right)}{\left|x_{u_{m}}(\xi, t)-x_{u_{m}}\left(\xi^{\prime}, t\right)\right|^{3}} J_{x_{u_{m}}\left(\xi^{\prime}, t\right)} d \xi^{\prime} \\
= & -\int_{\Omega} \frac{\nabla_{u_{m}} x_{u_{m}}(\xi, t) \cdot\left(\xi-\xi^{\prime}\right)\left(1+\int_{0}^{1} d s \int_{0}^{t} \partial_{s} u_{m}\left(\xi^{\prime}+s\left(\xi-\xi^{\prime}\right), \tau\right) d \tau\right)}{\left|\xi-\xi^{\prime}\right|^{3}\left|1+\int_{0}^{1} d s \int_{0}^{t} \partial_{s} u_{m}\left(\xi^{\prime}+s\left(\xi-\xi^{\prime}\right), \tau\right) d \tau\right|^{3}} \\
& \times J_{x_{u_{m}}\left(\xi^{\prime}, t\right)} d \xi^{\prime} .
\end{aligned}
$$

Assuming that

$$
\int_{0}^{t}\left\|u_{m \xi}(\cdot, \tau)\right\|_{L_{\infty}(\Omega)} d \tau<1
$$

we obtain

$$
\left\|\nabla_{u_{m}} U_{u_{m}}\right\|_{L_{r}\left(\Omega^{T}\right)} \leq \phi\left(a_{m}(T)\right) T^{1 / r}\left\|\int_{\Omega} \frac{d \xi^{\prime}}{\left|\xi-\xi^{\prime}\right|^{2}}\right\|_{L_{r}(\Omega)} \leq \phi\left(a_{m}(T)\right) T^{1 / r} .
$$

For simplicity we introduce

$$
X_{k}=\left\|u_{k}\right\|_{W_{r}^{2,1}\left(\Omega^{T}\right)}+\left\|q_{k}\right\|_{W_{r}^{1,0}\left(\Omega^{T}\right)}+\left\|q_{m}\right\|_{W_{r}^{1-1 / r, 1 / 2-1 /(2 r)}\left(S^{T}\right)} .
$$


Summing up the estimates for all terms of the r.h.s. of (3.5) we get

$$
\begin{aligned}
X_{m+1} \leq & a_{m} \phi\left(a_{m}\right) X_{m+1}+\phi\left(a_{m}\right) T^{r / 2-1} X_{m} X_{m+1} \\
& +\phi\left(a_{m}\right) a_{m} T^{1 / r}+\phi\left(a_{m}\right) T^{1 / 2+r / 2} X_{m}+\phi\left(a_{m}\right) X_{m} a_{m+1}(T) \\
& +\left(T^{1 / r}+T^{\beta}\right) \phi\left(a_{m}\right)+\phi\left(a_{m}(T)\right) T^{\beta} X_{m} .
\end{aligned}
$$

Putting

$$
a=\min \left\{\frac{r-1}{r}, \frac{r-2}{2}, \frac{1}{r}, \frac{1}{2}+\frac{r}{2}, \beta\right\}
$$

we have

$$
\begin{aligned}
X_{m+1} \leq & T^{a} \phi\left(a_{m}\right) X_{m} X_{m+1}+T^{a} \phi\left(a_{m}\right) X_{m} \\
& +\phi\left(a_{m}\right) T^{a} X_{m} X_{m+1}+T^{a} \phi\left(a_{m}\right) .
\end{aligned}
$$

By induction we prove that $X_{k} \leq 1\left(X_{0}=0\right)$. Taking $T$ such that $T \leq 1$ and $T^{a} \phi(1) \leq 1 / 4$, inserting $X_{m} \leq 1$ in (3.8), we obtain

$$
X_{m+1} \leq \frac{1}{4} X_{m+1}+\frac{1}{4}+\frac{1}{4} X_{m+1}+\frac{1}{4}
$$

which gives $X_{m+1} \leq 1$.

The proof of the lemma is complete.

Lemma 3.2. Assume that $S \in W_{r}^{2-1 / r}, v_{0} \in W_{r}^{2-2 / r}(\Omega)$. Then there exist $\bar{u} \in W_{r}^{2,1}\left(\Omega^{T}\right)$ and $\bar{p} \in W_{r}^{1,0}\left(\Omega^{T}\right) \cap W_{r}^{1-1 / r, 1 / 2-1 /(2 r)}\left(S^{T}\right)$ such that $u_{m} \rightarrow \bar{u}$ in $W_{r}^{2,1}\left(\Omega^{T}\right)$ and $q_{m} \rightarrow \bar{p}$ in $W_{r}^{1,0}\left(\Omega^{T}\right) \cap W_{r}^{1-1 / r, 1 / 2-1 /(2 r)}\left(S^{T}\right)$ as $m \rightarrow \infty$ for $T$ small enough.

Pro of. We show that $\left\{\left(u_{m}, q_{m}\right)\right\}_{n=1}^{\infty}$ is convergent. For this purpose we consider $v_{m}=u_{m+1}-u_{m}, r_{m}=q_{m+1}-q_{m}$ which satisfy the system

$$
\begin{array}{ll}
v_{m, t}-\operatorname{div} \mathbb{T}\left(v_{m}, r_{m}\right)=\operatorname{div}_{u_{m}} \mathbb{T}_{u_{m}}\left(u_{m+1}, q_{m+1}\right) & \\
\quad-\operatorname{div}_{u_{m-1}} \mathbb{T}_{u_{m-1}}\left(u_{m}, q_{m}\right)-\operatorname{div} \mathbb{T}\left(v_{m}, q_{m}\right) & \\
\quad+\nabla_{u_{m}} U_{u_{m}}-\nabla_{u_{m-1}} U_{u_{m-1}} \equiv I & \text { in } \Omega^{T}, \\
\operatorname{div} v_{m}=\operatorname{div} v_{m}-\operatorname{div}_{u_{m}} u_{m+1}+\operatorname{div}_{u_{m-1}} u_{m} \equiv J & \text { in } \Omega^{T}, \\
\bar{n}_{0} \cdot \mathbb{T}\left(v_{m}, r_{m}\right)=\bar{n}_{0} \mathbb{T}\left(v_{m}, r_{m}\right)-\bar{n}_{u_{m}} \mathbb{T}\left(u_{m+1}, q_{m+1}\right) & \\
\quad+\bar{n}_{u_{m-1}}\left(u_{m}, q_{m}\right)-p_{0} \bar{n}_{u_{m}}+p_{0} \bar{n}_{u_{m-1}} \equiv K & \text { on } S^{T}, \\
\left.v_{m}\right|_{t=0}=0 & \text { on } \Omega .
\end{array}
$$

By Theorem 1.2 we obtain an estimate on solutions of (3.9):

$$
\begin{aligned}
& \quad\left\|v_{m}\right\|_{W_{r}^{2,1}\left(\Omega^{T}\right)}+\left\|r_{m}\right\|_{W_{r}^{1,0}\left(\Omega^{T}\right)}+\left\|r_{m}\right\|_{W_{r}^{1-1 / r, 1 / 2-1 /(2 r)}\left(S^{T}\right)} \\
& \leq c\left(\|I\|_{L_{r}\left(\Omega^{T}\right)}+\|J\|_{W_{r}^{1,0}\left(\Omega^{T}\right)}+\|K\|_{W_{r}^{1-1 / r, 1 / 2-1 /(2 r)}\left(S^{T}\right)}+\|B\|_{L_{r}\left(\Omega^{T}\right)}\right),
\end{aligned}
$$

where $B$ is defined by the relation $J_{t}=\operatorname{div} B$. 
First we estimate the terms of the r.h.s. of $(3.9)_{1}$ in $L_{r}\left(\Omega^{T}\right)$. Let $I=$ $I_{1}+I_{2}$. We examine

$$
\begin{aligned}
I_{1}= & \operatorname{div}_{u_{m}} \mathbb{T}_{u_{m}}\left(u_{m+1}, q_{m+1}\right)-\operatorname{div}_{u_{m-1}} \mathbb{T}_{u_{m-1}}\left(u_{m}, q_{m}\right)-\operatorname{div} \mathbb{T}\left(v_{m}, q_{m}\right) \\
= & \left(\operatorname{div}_{u_{m}} \mathbb{T}_{u_{m}}-\operatorname{div} \mathbb{T}\right)\left(v_{m}, r_{m}\right) \\
& +\left(\operatorname{div}_{u_{m}} \mathbb{T}_{u_{m}}-\operatorname{div}_{u_{m-1}} \mathbb{T}_{u_{m-1}}\right)\left(u_{m}, q_{m}\right) \equiv I_{11}+I_{12} .
\end{aligned}
$$

$I_{11}$ is estimated in the same way as the first term of the r.h.s. of (3.5):

$$
\left\|I_{11}\right\|_{L_{r}\left(\Omega^{T}\right)} \leq \phi\left(a_{m}\right) a_{m}\left(\|v\|_{W_{r}^{2,1}\left(\Omega^{T}\right)}+\left\|r_{m}\right\|_{W_{r}^{1,0}\left(\Omega^{T}\right)}\right) .
$$

For the second term we have

$$
\begin{aligned}
\left\|I_{12}\right\|_{L_{r}\left(\Omega^{T}\right)}= & \|\left(\operatorname{div}_{u_{m}}\left(\mathbb{T}_{u_{m}}-\mathbb{T}_{u_{m-1}}\right)\right. \\
& \left.+\left(\operatorname{div}_{u_{m}}-\operatorname{div}_{u_{m-1}}\right) \mathbb{T}_{u_{m-1}}\right)\left(u_{m}, q_{m}\right) \|_{L_{r}\left(\Omega^{T}\right)} \\
\leq & \phi\left(a_{m}\right) T^{(r-1) / r}\left\|v_{m-1}\right\|_{W_{r}^{2,1}\left(\Omega^{T}\right)} .
\end{aligned}
$$

Next we consider

$$
\begin{aligned}
I_{2} & =\nabla_{u_{m}} U_{u_{m}}-\nabla_{u_{m-1}} U_{u_{m-1}} \\
& =\nabla_{u_{m}}\left(U_{u_{m}}-U_{u_{m-1}}\right)+\left(\nabla_{u_{m}}-\nabla_{u_{m-1}}\right) U_{u_{m-1}} \equiv I_{21}+I_{22} .
\end{aligned}
$$

The first term is

$$
\begin{aligned}
I_{21}= & -A\left(u_{m}\right) \int_{\Omega}\left(\frac{x_{m}(\xi, t)-y_{m}\left(\xi^{\prime}, t\right)}{\left|x_{m}(\xi, t)-y_{m}\left(\xi^{\prime}, t\right)\right|^{3}} J_{y_{m}\left(\xi^{\prime}, t\right)}\right. \\
& \left.-\frac{x_{m-1}(\xi, t)-y_{m-1}\left(\xi^{\prime}, t\right)}{\left|x_{m-1}(\xi, t)-y_{m-1}\left(\xi^{\prime}, t\right)\right|^{3}} J_{y_{m-1}\left(\xi^{\prime}, t\right)}\right) d \xi^{\prime} \\
= & -A\left(u_{m}\right) \int_{\Omega} \frac{x_{m}(\xi, t)-y_{m}\left(\xi^{\prime}, t\right)}{\left|x_{m}(\xi, t)-y_{m}\left(\xi^{\prime}, t\right)\right|^{3}}\left(J_{y_{m}\left(\xi^{\prime}, t\right)}-J_{y_{m-1}\left(\xi^{\prime}, t\right)}\right) d \xi^{\prime} \\
- & A\left(u_{m}\right) \int_{\Omega}\left(\frac{x_{m}(\xi, t)-y_{m}\left(\xi^{\prime}, t\right)}{\left|x_{m}(\xi, t)-y_{m}\left(\xi^{\prime}, t\right)\right|^{3}}\right. \\
& \left.-\frac{x_{m-1}(\xi, t)-y_{m-1}\left(\xi^{\prime}, t\right)}{\left|x_{m-1}(\xi, t)-y_{m-1}\left(\xi^{\prime}, t\right)\right|^{3}}\right) J_{y_{m-1}\left(\xi^{\prime}, t\right)} d \xi^{\prime} \\
\equiv & I_{211}+I_{212},
\end{aligned}
$$

where $x_{k}(\xi, t)=\xi+\int_{0}^{t} u_{k}\left(\xi, t^{\prime}\right) d t^{\prime}$ and $y_{k}\left(\xi^{\prime}, t\right)=\xi^{\prime}+\int_{0}^{t} u_{k}\left(\xi^{\prime}, t^{\prime}\right) d t^{\prime}$. Since

$$
\left|J_{y_{m}\left(\xi^{\prime}, t\right)}-J_{y_{m-1}\left(\xi^{\prime}, t\right)}\right| \leq c\left\|u_{m}-u_{m-1}\right\|_{W_{r}^{2,1}\left(\Omega^{T}\right)},
$$

we have

$$
\left\|I_{211}\right\|_{L_{r}\left(\Omega^{T}\right)} \leq \phi\left(a_{m}\right) T^{1 / r}\left\|v_{m-1}\right\|_{W_{r}^{2,1}\left(\Omega^{T}\right)} .
$$

The same holds for $I_{212}$. Since 


$$
\begin{aligned}
\int\left|\frac{x_{m}(\xi, t)-y_{m}\left(\xi^{\prime}, t\right)}{\left|x_{m}(\xi, t)-y_{m}\left(\xi^{\prime}, t\right)\right|^{3}}-\frac{x_{m-1}(\xi, t)-y_{m-1}\left(\xi^{\prime}, t\right)}{\left|x_{m-1}(\xi, t)-y_{m-1}\left(\xi^{\prime}, t\right)\right|^{3}}\right| d \xi^{\prime} \\
\leq c\left\|u_{m}-u_{m-1}\right\|_{W_{r}^{2,1}\left(\Omega^{T}\right)},
\end{aligned}
$$

we obtain

$$
\left\|I_{212}\right\|_{L_{r}\left(\Omega^{T}\right)} \leq \phi\left(a_{m}\right) T^{1 / r}\left\|v_{m-1}\right\|_{W_{r}^{2,1}\left(\Omega^{T}\right)} .
$$

We estimate $J$ in $W_{r}^{1,0}\left(\Omega^{T}\right)$ by the same quantity.

Let $K=K_{1}+K_{2}$, where

$$
\begin{aligned}
K_{1}= & \bar{n}_{0} \mathbb{T}\left(v_{m}, r_{m}\right)-\bar{n}_{u_{m}} \mathbb{T}\left(u_{m+1}, q_{m+1}\right)+\bar{n}_{u_{m-1}}\left(u_{m}, q_{m}\right) \\
= & \left(\bar{n}_{0} \mathbb{T}\left(v_{m}, r_{m}\right)-\bar{n}_{u_{m}} \mathbb{T}\left(v_{m}, r_{m}\right)\right) \\
& +\left(\bar{n}_{u_{m}} \mathbb{T}_{u_{m}}-\bar{n}_{u_{m-1}} \mathbb{T}_{u_{m-1}}\right)\left(u_{m}, q_{m}\right) \equiv K_{11}+K_{12}
\end{aligned}
$$

and

$$
K_{2}=-p_{0}\left(\bar{n}_{u_{m}}-\bar{n}_{u_{m-1}}\right) .
$$

The term $K_{11}$ is estimated just as the fourth term of the r.h.s. of (3.5):

$$
\begin{aligned}
\left\|K_{11}\right\|_{W_{r}^{1-1 / r, 1 / 2-1 /(2 r)}\left(S^{T}\right) \leq} & \phi\left(a_{m}(T)\right) a_{m}(T)\left\|v_{m}\right\|_{W_{r}^{2,1}\left(\Omega^{T}\right)} \\
& +\phi\left(a_{m}(T)\right) T^{r / 2-1}\left\|u_{m}\right\|_{W_{r}^{2,1}\left(\Omega^{T}\right)}\left\|v_{m}\right\|_{W_{r}^{2,1}\left(\Omega^{T}\right)} .
\end{aligned}
$$

The second term is

$K_{12}=\left(\bar{n}_{u_{m}}\left(\mathbb{T}_{u_{m}}-\mathbb{T}_{u_{m-1}}\right)+\left(\bar{n}_{u_{m}}-\bar{n}_{u_{m}-1}\right) \mathbb{T}_{u_{m-1}}\right)\left(u_{m}, q_{m}\right) \equiv K_{121}+K_{122}$.

For $K_{121}$ we have

$$
\left\|K_{121}\right\|_{W_{r}^{1-1 / r, 1 / 2-1 /(2 r)}\left(S^{T}\right)} \leq \phi\left(a_{m}(T)\right) T^{(r-1) / r}\left\|v_{m-1}\right\|_{W_{r}^{2,1}\left(\Omega^{T}\right)} .
$$

Since

$$
\left\|\bar{n}_{u_{m}}-\bar{n}_{u_{m-1}}\right\|_{W_{r}^{1-1 / r, 1 / 2-1 /(2 r)}\left(S^{T}\right)} \leq \phi\left(a_{m}(T)\right) T^{(r-1) / r}\left\|v_{m-1}\right\|_{W_{r}^{2,1}\left(\Omega^{T}\right)},
$$

we conclude that

$$
\begin{aligned}
\left\|K_{122}\right\|_{W_{r}^{1-1 / r, 1 / 2-1 /(2 r)}\left(S^{T}\right)}+\left\|K_{2}\right\|_{W_{r}^{1-1 / r, 1 / 2-1 /(2 r)}\left(S^{T}\right)} & \\
& \leq \phi\left(a_{m}(T)\right) T^{(r-1) / r}\left\|v_{m-1}\right\|_{W_{r}^{2,1}\left(\Omega^{T}\right)} .
\end{aligned}
$$

Finally we have to examine $B$ which is defined by $J_{t}=\operatorname{div} B$. We have

$$
\begin{aligned}
J & =\operatorname{div} v_{m}-\operatorname{div}_{u_{m}} u_{m-1}+\operatorname{div}_{u_{m-1}} u_{m} \\
& =\left(\operatorname{div} v_{m}-\operatorname{div}_{u_{m}} v_{m}\right)+\left(\operatorname{div}_{u_{m-1}} u_{m}-\operatorname{div}_{u_{m}} u_{m}\right) \equiv J_{1}+J_{2} .
\end{aligned}
$$

To examine $J_{1}$ we proceed as in the case of the seventh term of the r.h.s. of (3.5). By the same argument as in Lemma 3.1 we have

$$
J_{2}=\left(\operatorname{div}_{u_{m-1}}-\operatorname{div}_{u_{m}}\right) u_{m}=\operatorname{div} \cdot\left(\left(A_{m-1}^{*}-A_{m}^{*}\right) u_{m}\right) .
$$

Hence we put $B_{2}=\left(\left(A_{m-1}^{*}-A_{m}^{*}\right) u_{m}\right)_{t}$ and we get, just as for $N_{2}$ in the proof of Lemma 3.1, the following estimate: 


$$
\left\|B_{2}\right\|_{L_{r}\left(\Omega^{T}\right)} \leq \phi\left(a_{m}(T)\right)\left(T^{(r-1) / r}+T^{\beta}\right)\left\|v_{m-1}\right\|_{W_{r}^{2,1}\left(\Omega^{T}\right)},
$$

where $0<\beta<1 / 2-1 / r$.

Define

$$
Y_{m}=\left\|v_{m}\right\|_{W_{r}^{2,1}\left(\Omega^{T}\right)}+\left\|r_{m}\right\|_{W_{r}^{1,0}\left(\Omega^{T}\right)}+\left\|r_{m}\right\|_{W_{r}^{1-1 / r, 1 / 2-1 /(2 r)}\left(S^{T}\right)} .
$$

Summing up the estimates for all terms of the r.h.s. of (3.10) we obtain

$$
Y_{m} \leq \phi\left(a_{m}(T)\right)\left(T^{(r-1) / r}+T^{r / 2-1}+T^{\beta}\right) Y_{m}+\phi\left(a_{m}(T)\right)\left(T^{(r-1) / r}+T^{\beta}\right) Y_{m-1} \text {. }
$$

Taking $T$ so small that $\phi\left(a_{m}(T)\right)\left(T^{(r-1) / r}+T^{r / 2-1}+T^{\beta}\right) \leq 1 / 2$ we get

$$
Y_{m} \leq \phi\left(a_{m}(T)\right)\left(T^{(r-1) / r}+T^{\beta}\right) Y_{m-1} .
$$

Thus if $\phi\left(a_{m}(T)\right)\left(T^{(r-1) / r}+T^{\beta}\right)<1$ we have a contraction, hence $Y_{m} \rightarrow 0$ as $m \rightarrow \infty$. This yields the existence of $\bar{u} \in W_{r}^{2,1}\left(\Omega^{T}\right)$ and $\bar{p} \in W_{r}^{1,0}\left(\Omega^{T}\right) \cap$ $W_{r}^{1-1 / r, 1 / 2-1 /(2 r)}\left(S^{T}\right)$ such that

$$
\begin{array}{ll}
u_{m} \rightarrow \bar{u} & \text { in } W_{r}^{2,1}\left(\Omega^{T}\right), \\
q_{m} \rightarrow \bar{p} & \text { in } W_{r}^{1,0}\left(\Omega^{T}\right) \cap W_{r}^{1-1 / r, 1 / 2-1 /(2 r)}\left(S^{T}\right) .
\end{array}
$$

The proof of the lemma is complete.

By Lemma 3.2 we see that system (3.1) has a unique solution $(u, q)$ in $W_{r}^{2,1}\left(\Omega^{T}\right) \times W_{r}^{1,0}\left(\Omega^{T}\right) \cap W_{r}^{1-1 / r, 1 / 2-1 /(2 r)}\left(S^{T}\right)$. By Lemma 3.1 we get the estimate

$$
\begin{aligned}
\|u\|_{W_{r}^{2,1}\left(\Omega^{T}\right)}+\|q\|_{W_{r}^{1,0}\left(\Omega^{T}\right)}+\|q\|_{W_{r}^{1-1 / r, 1 / 2-1 /(2 r)}\left(S^{T}\right)} & \\
& \leq c\left(\left\|v_{0}\right\|_{W_{r}^{2-2 / r}(\Omega)},\|S\|_{W_{r}^{2-2 / r}}\right) .
\end{aligned}
$$

Since for $u \in W_{r}^{2,1}\left(\Omega^{T}\right)$ the transformation (1.7) is invertible, from (3.11) we obtain estimate (1.8). Theorem 1.1 is proved.

\section{References}

[1] O. V. Besov, V. P. Il'in and S. M. Nikol'skiŭ, Integral Representations of Functions and Imbedding Theorems, Nauka, Moscow, 1975 (in Russian).

[2] O. A. Ladyzhenskaya, V. A. Solonnikov and N. N. Ural'tseva, Linear and Quasilinear Equations of Parabolic Type, Amer. Math. Soc., Providence, RI, 1975.

[3] P. B. Mucha and W. M. Zaj,aczkowski, On the existence for the Cauchy-Neumann problem for the Stokes system in the $L_{p}$-framework, Studia Math., to appear.

[4] V. A. Solonnikov, On nonstationary motion of an isolated volume of a viscous incompressible fluid, Izv. Akad. Nauk SSSR 51 (1987), 1065-1087 (in Russian). 
[5] V. A. Solonnikov, Solvability on a finite time interval of the problem of evolution of a viscous incompressible fluid bounded by a free surface, Algebra Anal. 3 (1991), 222-257 (in Russian).

Piotr Bogusław Mucha

Institute of Applied

Mathematics and Mechanics

Warsaw University

Banacha 2

02-097 Warszawa, Poland

E-mail: mucha@hydra.mimuw.edu.pl
Wojciech Zajączkowski Institute of Mathematics Polish Academy of Sciences Śniadeckich 8 00-950 Warszawa, Poland and

Institute of Mathematics and Operations Research Military University of Technology Kaliskiego 2 01-489 Warszawa, Poland E-mail: wz@impan.gov.pl

Received on 28.5.1999;

revised version on 17.1.2000 